\section{Magnitude of the Mueller-Lyer illusion as a function of hue, saturation, and fundus pigmentation}

\author{
PAMELA C. EBERT and ROBERT H. POLLACK \\ University of Georgia, Athens, Ga. 30601
}

One hundred Ss, 50 male and 50 female undergraduate students, were tested to determine the effects of saturation, hue, and fundus pigmentation on the magnitude of the Mueller-Lyer illusion. It was found that the highly saturated (chroma 12) red figure produced a smaller illusion than the unsaturated (chroma 6) red or blue figures. Only the magnitude of the illusion produced by the highly saturated yellow figure correlated significantly with the lightness of fundus pigmentation.

In two previous studies (Pollack, $1970 \mathrm{a}, \mathrm{b})$ it was found that the magnitude of the Mueller-Lyer illusion varied as a function of hue when lightness contrast was absent. With relatively unsaturated samples (chroma 6), red produced the largest illusion, followed by blue, yellow, and green, in that order. Pollack (1970b) also showed that while the magnitude of illusion of a white figure on a black ground decreased as a function of age, chromatic figures on a gray ground of equal lightness showed no such decline. Since gross differences in fundus pigmentation (Pollack \& Silvar, 1967) had also been shown to be related to illusion magnitude in preadolescent boys, it was decided to correlate illusion magnitude in this study with lightness of fundus pigmentation to see whether or not the relation could be demonstrated for chromatic stimuli in adults.

\section{SUBJECTS}

The Ss were 50 male and 50 female white undergraduate students ranging in age from 18 to 25 years, all having uncorrected acuities of $20 / 20$ or better. None showed color defects as measured by the AO Pseudoisochromatic Plates. \section{APPARATUS}

Each stimulus consisted of the open component (forked ends connected by a straight line) of the Mueller-Lyer figure and a straight unbounded comparison line. All lines were $1 \mathrm{~mm}$ thick. The oblique-inducing lines were $5 \mathrm{~mm}$ long, and the standard line between the obliques was $22 \mathrm{~mm}$. The comparison lines varied in $.5-\mathrm{mm}$ steps from $20 \mathrm{~mm}$ through $29 \mathrm{~mm}$. Each comparison line was mounted on a 35-mm square field consisting of Munsell gray paper. The standard illusion figure was also mounted on each slide. Both figures were vertical and placed symmetrically, one $5 \mathrm{~mm}$ to the left and one $5 \mathrm{~mm}$ to the right of a central fixation point. The maximal visual angle was $1 \mathrm{deg} 7 \mathrm{~min}$ in the vertical dimension.

The figures were constructed of Munsell papers; the spectral hues differed from their grounds in saturation only. Two sets of three series of figures were used. The unsaturated figures were $5 \mathrm{R} 5 / 6$, $5 Y 5 / 6$, and $5 \mathrm{~B} 5 / 6$ on N5/gray. The saturated figures were $5 \mathrm{R} 5 / 12,5 \mathrm{~B} 5 / 12$ on N5/gray, and 5 Y5/12 on N8/gray. Overall reflectance of the yellow figure was reduced by a .3 neutral density filter to equal other figures.

Measures of fundus reflectance were obtained for each $S$ in the high-saturation group, using a highly sensitive Photovolt light meter connected to one eyepiece of a Bausch and Lomb binocular ophthalmoscope. Readings were taken from a point on the retina just to the left of the foveal depression.

\section{PROCEDURE}

The low-saturation condition was run first with 25 males and 25 females. The figures were presented tachistoscopically for $1,500 \mathrm{msec}$ at a viewing distance of $1,500 \mathrm{~mm}$ in illuminant $C$. The order of presentation of the hues was randomized across $\mathrm{Ss}$, and left or right location of the standard was counterbalanced.

A converging method of limits was used. Six trials were used for each hue. The same procedure was repeated 1 year later with the remaining $50 \mathrm{Ss}$, with a measure of fundus pigmentation also taken.

\section{RESULTS}

PSEs were obtained for each trial, and the mean PSE across trials was calculated for each S. Since earlier results (Pollack, 1970a) indicated no sex differences in illusion scores, this dimension was ignored. The data were analyzed by saturation level and across hues, using a mixed-model ANOVA for repeated measures across hues and independence for saturation levels
(McNemar, 1969). No significant main effect for saturation was found; the hue effect $(F=2.5, \quad \mathrm{df}=2,196$, $p<.01$ ) was found for the Hue by $S$ at uration interaction. A Newman-Keuls test for paired comparisons of the Hue by Saturation interaction revealed that the highly saturated red figure produced a smaller illusion than the unsaturated red or blue figures. It was also found that with low saturation, a larger illusion was produced by red than by blue and larger by blue than by yellow, while the order was reversed for high saturation (see Fig. 1).

Product-moment correlations between fundus pigmentation lightness and illusion magnitude of the highly saturated hues were calculated. Only the magnitude of the illusion produced by the yellow figure correlated significantly with the lightness of fundus pigmentation $(r=.29, \mathrm{df}=48$, $p<.05)$. The other correlations were negligible $(r=.09$, for both red and blue). The intercorrelations among hues, however, were all significant, indicating the commonality of the illusion tasks. \section{DISCUSSION}

Wickelgren (1965) found that the lightness contrast level of the figure's inducing lines influenced the magnitude of illusion as a function of the amount of contrast. The results of the present study, in which hue contrast was varied in the absence of lightness contrast, demonstrated quite different results. In the case of hue contrast alone, the mechanism must be somewhat different, since saturation increased only the magnitude of the yellow figure while it decreased that of the red.

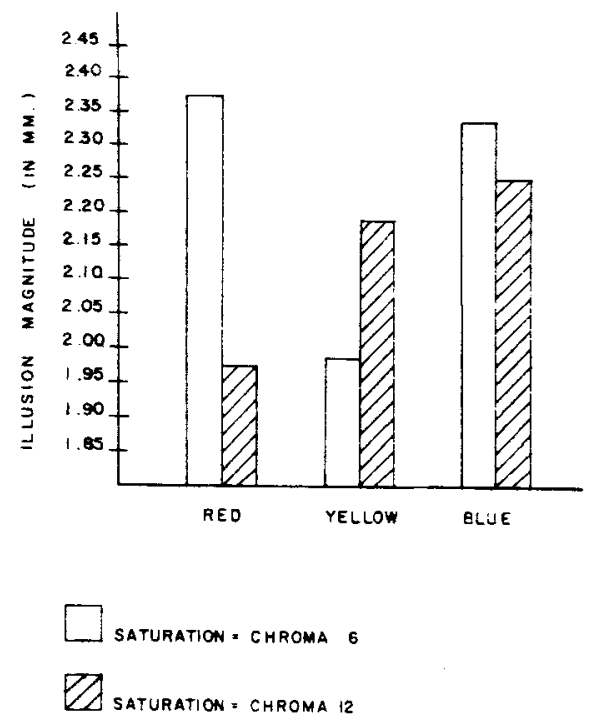

Fig. 1. Ilusion magnitude as a function of hue and saturation. 
It has been our experience that a yellow figure on a ground of equal lightness does not stand out well. The increase of saturation and thus the increase of hue contrast might be expected to improve the configural qualities of yellow and thus increase the illusory effects of the inducing lines. Just why the opposite occurred for the red figure is a mystery to be attacked by future research.

Our results may be related to recent work by De Valois (1970) which deals with the lateral geniculate responses to contour with chromatic and achromatic stimuli. Achromatic stimuli produce maximal responses at their edges, while chromatic stimuli produce maximal responses at their centers. Furthermore, those cells which are most responsive to hue are less responsive to intensity and intensity differences than those which respond to foveal achromatic light. Yellow is the most sensitive of those, followed by blue, green, and red.

Overall, the results tend to confirm the contention (Pollack, 1970b) that the processes underlying the contour effects of figures produced by hue contrast alone are different from those underlying lightness contrast alone.

$$
\text { REFERENCES }
$$

DeVALOIS, R. L. Analysis of brightness and color by primate visual systems. Invited address, American Psychological Association, Miami, 1970.

MCNEMAR, Q. Psychological statistics. 4th ed. New Yodk: Wiley, 1969.

POLLACK, R. H. Magnitude of the Mueller-L yer illusion as a function of hue in the absence of lightness contrast. Proceedings of the American Psychological Association, 1970a, 53-54.

POLLACK, R. H. Mueller-Lyer illusion: Effect of age, lightness contrast and hue. Science, $1970 \mathrm{~b}, 170,93-95$.

POLLACK, R. H., \& SILVAR, S. Magnitude of the Mueller-Lyer illusion in children as a function of pigmentation of the Fundus oculi. Psychonomic Science, 1967, 8, 2, 83-84.

WTKELGREN, G. G. Brightness contrast and length perception in the Mueller-Lyer illusion. Vision Research, 1965, 5 , 141-150. 